\title{
Association between the intake of $\alpha$-linolenic acid and the risk of CHD
}

Mia Sadowa Vedtofte ${ }^{1 *}$, Marianne U. Jakobsen ${ }^{2}$, Lotte Lauritzen ${ }^{3}$, Eilis J. O'Reilly ${ }^{4}$, Jarmo Virtamo $^{5}$, Paul Knekt ${ }^{5}$, Graham Colditz ${ }^{6}$, Göran Hallmans ${ }^{7}$, Julie Buring ${ }^{8}$, Lyn M. Steffen 9 , Kimberly Robien ${ }^{10,11}$, Eric B. Rimm ${ }^{4}$ and Berit L. Heitmann ${ }^{1,12}$

${ }^{1}$ Research Unit for Dietary Studies, Institute of Preventive Medicine, Copenhagen Municipal Hospitals Region H, Copenhagen, Denmark.

${ }^{2}$ Section for Epidemiology, Department of Public Health, Aarbus University, Aarbus, Denmark

${ }^{3}$ Department of Nutrition, Exercise and Sports, Faculty of Science, University of Copenhagen, Frederiksberg, Denmark

${ }^{4}$ Departments of Nutrition and Epidemiology, Harvard School of Public Health, Boston, MA, USA

${ }^{5}$ Department of Chronic Disease Prevention, National Institute for Health and Welfare, Helsinki, Finland

${ }^{6}$ Department of Surgery, Washington University School of Medicine, St Louis, MO, USA

${ }^{7}$ Department of Public Health and Clinical Medicine, Nutritional Research, Umeå University, Umeå, Sweden

${ }^{8}$ Division of Preventive Medicine, Department of Medicine, Brigham and Women's Hospital, Boston, MA, USA

${ }^{9}$ Division of Epidemiology and Community Health, University of Minnesota School of Public Health, Minneapolis, MN, USA

${ }^{10}$ Department of Epidemiology and Biostatistics, School of Public Health and Health Services, George Washington University, Washington, DC, USA

${ }^{11}$ Department of Exercise Science, School of Public Health and Health Services, George Washington University, Washington, DC, USA

${ }^{12}$ National Institute of Public Health, University of Southern Denmark, Copenhagen, Denmark

(Submitted 7 November 2013 - Final revision received 5 February 2014 - Accepted 10 February 2014 - First published online 25 June 2014)

\section{Abstract}

The intake of the mainly plant-derived $n$-3 PUFA $\alpha$-linolenic acid (ALA) has been reported to be associated with a lower risk of CHD. However, the results have been inconsistent. Therefore, the objective of the present study was to examine the association between the intake of ALA and the risk of CHD. Potential effect modification by the intake of long-chain $n-3$ PUFA ( $n$ - 3 LCPUFA) was also investigated. Data from eight American and European prospective cohort studies including 148675 women and 80368 men were used. The outcome measure was incident CHD (CHD event and death). During 4-10 years of follow-up, 4493 CHD events and 1751 CHD deaths occurred. Among men, an inverse association (not significant) between the intake of ALA and the risk of CHD events and deaths was observed. For each additional gram of ALA consumed, a $15 \%$ lower risk of CHD events (hazard ratios (HR) $0.85,95 \%$ CI $0 \cdot 72,1 \cdot 01$ ) and a $23 \%$ lower risk of CHD deaths (HR $0 \cdot 77,95 \%$ CI $0 \cdot 58,1 \cdot 01)$ were observed. No consistent association was observed among women. No effect modification by the intake of $n$-3 LCPUFA was observed.

Key words: $\alpha$-Linolenic acid: CHD: Epidemiology

CHD is the leading cause of morbidity and mortality in the world ${ }^{(1)}$. CHD arises from genetic and modifiable lifestyle factors including diet. One of the important dietary components identified is the low intake of $n-3$ PUFA. The marine productderived long-chain $n$-3 PUFA ( $n$ - 3 LCPUFA), EPA and DHA, have for long been the main focus of research with regard to the importance of $n-3$ PUFA in relation to CHD. Since the pioneering studies carried out among Greenland Eskimos have suggested that high intakes of EPA and DHA can protect against $\mathrm{CHD}^{(2)}$, many studies and meta-analyses have confirmed this association ${ }^{(3-7)}$. However, the long-term association between the intake of the mainly plant-derived $n-3$ PUFA $\alpha$-linolenic acid (ALA) and the risk of CHD is not clear ${ }^{(8-11)}$. A recent meta-analysis of three prospective studies carried out in 2012 has suggested that each $1 \mathrm{~g} / \mathrm{d}$ of ALA intake is associated with a $20 \%$ lower risk of CHD events ${ }^{(11)}$.

Abbreviations: ALA, $\alpha$-linolenic acid; ARIC, Atherosclerosis Risk in Communities Study; ATBC, Alpha-Tocopherol, Beta-Carotene Cancer Prevention Study; FMC, Finnish Mobile Clinic Health Examination Survey; HPFS, Health Professionals Follow-up Study; HR, hazard ratio; IIHD, Israeli Ischemic Heart Disease Study; IWHS, Iowa Women's Health Study; LA, linoleic acid; $n$-3 LCPUFA, long-chain $n$-3 PUFA; NHS, Nurses' Health Study; VIP, Västerbotten Intervention Program; WHS, Women's Health Study.

*Corresponding author: M. S. Vedtofte, fax +453816 31 19, email mia.sadowa.vedtofte@ regionh.dk 
The intake of fish and other marine products is limited in many countries, and ALA primarily from plant oils and nuts (e.g. rapeseed oils, walnuts and soyabean) is the predominant source of $n$-3 PUFA in the typical Western diet. In the human body, ALA is converted into $n$ - 3 LCPUFA by desaturation and elongation, but this conversion is limited and generally much lower in men than in women ${ }^{(12,13)}$. The effect of ALA intake on the risk of CHD may be influenced by $n-3$ LCPUFA, because they, via feedback inhibition of the activity of $\Delta 6$ desaturase (the key limiting enzyme), regulate the conversion of ALA into $n$ - 3 LCPUFA. Therefore, it is possible that ALA may particularly reduce the risk of CHD among individuals with a low intake of $n$-3 LCPUFA $^{(14)}$.

In the present study, we investigated the association between ALA intake and CHD risk in populations consuming different diets and exhibiting a wide range of ALA intakes, using data from eight American and European prospective cohort studies included in the Pooling Project of Cohort Studies on Diet and Coronary Disease. We hypothesised that the intake of ALA was inversely associated with the risk of CHD and that the inverse association between the intake of ALA and the risk of CHD was stronger in subjects with a low intake of $n-3$ LCPUFA than in those with a high intake of $n-3$ LCPUFA.

\section{Subjects and methods}

\section{Study population}

A total of eleven prospective cohort studies (the Adventist Health Study ${ }^{(15)}$, the Atherosclerosis Risk in Communities Study (ARIC) ${ }^{(16)}$, the Alpha-Tocopherol, Beta-Carotene Cancer Prevention Study (ATBC) ${ }^{(17)}$, the Finnish Mobile Clinic Health Examination Survey (FMC) ${ }^{(18)}$, the Glostrup Population Study ${ }^{(19)}$, the Health Professionals Follow-up Study (HPFS) ${ }^{(20)}$, the Israeli Ischemic Heart Disease Study (IIHD) ${ }^{(21)}$, the Iowa Women's Health Study (IWHS) ${ }^{(22)}$, the Nurses' Health Study (NHS) ${ }^{(23)}$, the Västerbotten Intervention Program (VIP) ${ }^{(24)}$, and the Women's Health Study (WHS) ${ }^{(25)}$ ) met the following criteria and were included in the Pooling Project of Cohort Studies on Diet and Coronary Disease: (1) a published prospective study with at least 150 incident CHD cases; (2) a study determining usual dietary intake using a FFQ, a dietary history interview, or a $7 \mathrm{~d}$ weighed food record at baseline; (3) a validation or a repeatability study of the dietary intake assessment method. Among these studies, three (the Adventist Health Study, the Glostrup Population Study and the IIHD) were excluded from the present analysis due to lack of information on ALA intake. The characteristics of the remaining eight studies, five carried out in the USA, two in Finland and one in Sweden, are summarised in Table 1. From the cohorts, we excluded individuals who were aged $<35$ years, had a history of CVD, diabetes, or cancer (except non-melanoma skin cancer), and had an extreme energy intake, i.e. intakes greater or less than three standard deviations from the study-specific log-transformed mean energy intake of the baseline populations. The final study population consisted of 229043 persons ( $65 \%$ women).

\section{Habitual dietary intake determination}

Habitual dietary intake was determined at baseline using a FFQ in seven of the cohort studies and by a dietary history interview in one cohort study (Table 1). The FFQ that were used in the ARIC, the NHS, the HPFS, the WHS and the IWHS were similar or a modified version of the original sixty-one-item Willett FFQ developed in $1980^{(23)}$. However, as the FFQ were modified, the number of items in each study differed; the FFQ used in the ARIC, the HPFS, the WHS and the IWHS contained 66, 131, 131 and 116 items, respectively. As the ARIC used the oldest version of the FFQ with only sixty-six items, it did not have the same details on, for example, cooking oil. In the NHS carried out in 1980, a sixty-one-item FFQ was used to assess dietary intake. The questionnaire was expanded to 116 items in 1984 and administered in 1986 and 1990. The data from the NHS used in the present study were those obtained in 1986. The participants were asked to report how often, on average, they had consumed given amounts of the various specified foods during the previous year. The average daily intake of nutrients was then computed by multiplying the frequency of intake by the nutrient composition of the specified portion size. The composition values of ALA (and other nutrients) were obtained from the Harvard University food composition database and the US Department of Agriculture ${ }^{(26)}$. The Finnish FFQ used in the ATBC included 276 food items, and the participants were asked to report their usual intake and portion size of foods during the previous year ${ }^{(17,27)}$. The type of fat used on bread, which is an important source of fat in the Finnish diet, was specified by the brand name of the product ${ }^{(28)}$. The fatty acid content of the foods was based on analyses of seventy-seven fatty acids present in Finnish foods carried out at the Department of Food Chemistry, University of Helsinki ${ }^{(28)}$. The questionnaire used in the VIP ${ }^{(24)}$ included eighty-four food items. The subjects were asked to report their habitual dietary intake during the previous year and typical portion sizes were indicated using colour photographs. The average daily intake was calculated by multiplying the frequency of intake by a portion size value using the Swedish national food composition database $^{(24)}$. The FMC used a dietary history interview, where the interviewer asked the participants about their habitual dietary intake during the previous year ${ }^{(29)}$. The subjects reported their food intake on a daily, weekly or monthly basis. The ingredients of mixed foods were broken down into components using a recipe file and the intake of fruits and berries, vegetables, and cereals as well as the subgroups of these food categories and separate food items such as dairy and meat products was calculated per $d$. The total energy intake was calculated from the intake of protein, fat and available carbohydrates according to the Finnish food composition tables ${ }^{(29)}$.

\section{Outcome}

The outcome of interest was incident CHD events (fatal and non-fatal). Because only the IWHS had self-reported data on incident CHD, we only used data on fatal CHD events from this study. All studies used standardised criteria to define non-fatal and fatal CHD cases $^{(30)}$. 
Table 1. Characteristics of the cohort studies included in the Pooling Project of Cohort Studies on Diet and Coronary Disease (Median values and $80 \%$ central ranges)

\begin{tabular}{|c|c|c|c|c|c|c|c|c|c|c|c|c|c|c|}
\hline \multirow[b]{3}{*}{ Study } & \multirow[b]{3}{*}{ Country } & \multirow[b]{3}{*}{$\begin{array}{l}\text { Year of } \\
\text { inception }\end{array}$} & \multirow[b]{3}{*}{$\begin{array}{c}\text { Baseline } \\
\text { cohort sample } \\
\text { size }^{*}\end{array}$} & \multirow{2}{*}{\multicolumn{2}{|c|}{$\begin{array}{l}\text { Age at baseline } \\
\text { (years) }\end{array}$}} & \multirow{2}{*}{\multicolumn{2}{|c|}{$\begin{array}{l}\text { Median follow-up } \\
\text { time }\end{array}$}} & \multirow{2}{*}{\multicolumn{2}{|c|}{$\begin{array}{c}\text { Number of CHD } \\
\text { events }\end{array}$}} & \multirow[b]{3}{*}{$\begin{array}{l}\text { Dietary intake } \\
\text { assessment } \\
\text { method }\end{array}$} & \multicolumn{4}{|c|}{ Dietary PUFA intake (energy adjusted, g/d) } \\
\hline & & & & & & & & & & & \multicolumn{2}{|c|}{ ALA } & \multicolumn{2}{|c|}{$\mathrm{EPA}+\mathrm{DHA}$} \\
\hline & & & & Median & $\begin{array}{l}80 \% \\
\text { Central } \\
\text { range }\end{array}$ & $\begin{array}{l}\text { Total } \\
\text { CHD } \\
\text { events }\end{array}$ & $\begin{array}{l}\text { CHD } \\
\text { deaths }\end{array}$ & $\begin{array}{l}\text { Total } \\
\text { CHD } \\
\text { events }\end{array}$ & $\begin{array}{l}\text { CHD } \\
\text { deaths }\end{array}$ & & Median & $\begin{array}{l}80 \% \\
\text { Central } \\
\text { range }\end{array}$ & Median & $\begin{array}{l}80 \% \\
\text { Central } \\
\text { range }\end{array}$ \\
\hline \multicolumn{15}{|l|}{ Women } \\
\hline ARIC & USA & 1987 & 6481 & 53 & $47-62$ & 9.2 & - & 123 & _- & FFQ & 0.70 & $0.54-0.90$ & 0.21 & $0.05-0.62$ \\
\hline FMC & Finland & 1966 & 2481 & 49 & $38-65$ & $10 \cdot 0$ & $10 \cdot 0$ & 162 & 48 & $\mathrm{DH}$ & 0.53 & $0.42-0.87$ & $0 \cdot 15$ & $0.06-0.36$ \\
\hline IWHS & USA & 1986 & 30180 & 61 & $56-67$ & - & $10 \cdot 0$ & - & 294 & FFQ & 0.94 & $0.71-1.25$ & 0.13 & $0.02-0.35$ \\
\hline NHS & USA & 1986 & 61706 & 52 & $43-62$ & $10 \cdot 0$ & $10 \cdot 0$ & 696 & 208 & $\mathrm{FFQ}$ & 0.97 & $0.72-1.33$ & 0.18 & $0.06-0.42$ \\
\hline VIP & Sweden & 1992 & 10555 & 50 & $40-60$ & 4.1 & - & 23 & - & FFQ & 1.26 & $0.96-1.81$ & 0.10 & $0.03-0.23$ \\
\hline WHS & USA & 1993 & 37272 & 52 & $46-64$ & $5 \cdot 3$ & $5 \cdot 3$ & 152 & 10 & $\mathrm{FFQ}$ & 0.92 & $0.69-1.26$ & 0.15 & $0.05-0.36$ \\
\hline \multicolumn{15}{|l|}{ Men } \\
\hline ARIC & USA & 1987 & 5240 & 54 & $47-63$ & 9.2 & 9.2 & 269 & 51 & FFQ & 0.93 & $0.72-1.20$ & 0.20 & $0.03-0.62$ \\
\hline ATBC & Finland & 1985 & 21141 & 57 & $51-65$ & $6 \cdot 0$ & $6 \cdot 1$ & 1339 & 534 & FFQ & 1.20 & $0.74-1.92$ & 0.34 & $0.16-0.66$ \\
\hline FMC & Finland & 1966 & 2712 & 47 & $37-63$ & $10 \cdot 0$ & $10 \cdot 0$ & 322 & 147 & $\mathrm{DH}$ & 0.68 & $0.55-0.98$ & 0.23 & $0.09-0.59$ \\
\hline HPFS & USA & 1986 & 41754 & 53 & $42-67$ & $9 \cdot 7$ & $9 \cdot 7$ & 1273 & 421 & FFQ & $1 \cdot 12$ & $0.83-1.54$ & 0.26 & $0.07-0.58$ \\
\hline VIP & Sweden & 1992 & 9521 & 50 & $40-60$ & $4 \cdot 1$ & $4 \cdot 1$ & 134 & 38 & FFQ & 1.73 & $1.27-2.51$ & 0.12 & $0.03-0.30$ \\
\hline Women, total & & & 148675 & & & & & 1156 & 560 & & 1.01 & $0.58-1.64$ & 0.15 & $0.04-0.42$ \\
\hline Men, total & & & 80368 & & & & & 3337 & 1191 & & $1 \cdot 17$ & $0.64-1.62$ & 0.26 & $0.06-0.66$ \\
\hline Total & & & 229043 & & & & & 4493 & 1751 & & 1.06 & $0.60-1.06$ & 0.19 & $0.05-0.50$ \\
\hline
\end{tabular}

ALA, $\alpha$-linolenic acid; ARIC, Atherosclerosis Risk in Communities Study; FMC, Finnish Mobile Clinic Health Examination Survey; DH, dietary history interview; IWHS, Iowa Women's Health Study; NHS, Nurses' Health Study; VIP, Västerbotten Intervention Program; WHS, Women's Health Study; ATBC, Alpha-Tocopherol, Beta-Carotene Cancer Prevention Study; HPFS, Health Professionals Follow-up Study.

* Sample size after the exclusion of subjects with a baseline age $<35$ years, baseline CVD, cancers, and diabetes mellitus, very high or low reported energy intake and missing information on dietary PUFA intake. 


\section{Statistical analyses}

Within each study, hazard ratios (HR) with 95\% CI for the incidence of fatal and non-fatal CHD events were calculated using Cox proportional hazards regression with follow-up duration in the study (years) as the time metric ${ }^{(31)}$. The observation time for each participant was defined as the date on which information on dietary intake was obtained and until the occurrence of CHD, death from another cause, disappearance or end of the follow-up, whichever came first. Studies with follow-up periods of more than 10 years were truncated to reduce possible effect modification by time. The analyses were carried out separately for men and women. The intake of ALA was adjusted for total energy using the residual method and modelled as a continuous variable followed by analyses with ALA intake as a categorical variable, in quintiles, and also as a binary variable (below/above the median). Subsequently, to assess possible effect modification by the intake of $n$-3 LCPUFA, we stratified analyses with ALA and $n$-3 LCPUFA intakes using combined categories of higher ( $>$ median) $v$. lower $(<$ median) intake. Furthermore, analyses were carried out to explore possible age-related differences in the association between ALA intake and CHD risk. The study population was divided into two age groups in further analyses: those aged $<60$ years at baseline and those aged $\geq 60$ years at baseline. The study-specific logs of HR were weighted by the inverse of their variances, and a pooled (combined) estimate of the HR was computed using a random-effects model. Evidence for between-study heterogeneity among the study-specific HR was examined using the estimated between-study variance component $Q$ statistic $^{(31)}$.

The following potential confounders were included in the statistical models: BMI $\left(<23,23-25,25-30\right.$, or $\left.>30 \mathrm{~kg} / \mathrm{m}^{2}\right)$; highest attained educational level ( $<$ high school, high school, or $>$ high school); smoking habits (non-, ex-, and current-smokers and in the last category 1-4, 5-14, 15-24, or $\geq 25$ cigarettes/d); physical activity (five levels); alcohol intake $(0,0$ to $<5,5$ to $<10,10$ to $<15,15$ to $<30,30$ to $<50$, or $>50 \mathrm{~g} / \mathrm{d}$ ); total energy intake (the sum of intakes of energy derived from fat, carbohydrates and protein in $\mathrm{kJ} / \mathrm{d}$ );
SFA, trans-fatty acid, MUFA, linoleic acid (LA), and n-3 LCPUFA intake in $\mathrm{g} / \mathrm{d}$; quintiles of dietary fibre intake; history of hypertension (yes or no). The association between the intake of ALA and the risk of CHD was investigated using three models. Model 1 included energy-adjusted intake of ALA in $\mathrm{g} / \mathrm{d}$ and age at baseline (years), as well as the calendar year in which the baseline diet questionnaire was returned. The variables were entered into the model through the STRATA statement. Model 2 included the variables of model 1 and the following known risk factors for CHD: BMI; educational level; smoking habits; physical activity; history of hypertension. Model 3 included the variables of model 2 and the following dietary risk factors: alcohol intake; total energy intake; quintiles of fibre intake; trans-fatty acid, SFA, MUFA, LA, and $n-3$ LCPUFA intake. We tested ALA intake for nonlinearity by including an energy-adjusted ALA variable squared to the model and pooled the effect estimate for this term. The analyses were carried out using SAS 9.2 statistical software (SAS Institute, Inc.).

\section{Results}

The characteristics of the cohort studies are given in Table 1. During 4-10 years of follow-up, 4493 CHD events and 1751 CHD deaths occurred among the 229043 subjects. The 10th, 50th and 90th percentiles of energy-adjusted intake of ALA were $0.58,1.01$ and $1.64 \mathrm{~g} / \mathrm{d}$ in women, respectively, and $0.64,1.17$ and $1.62 \mathrm{~g} / \mathrm{d}$ in men, respectively. The intake of ALA was modestly correlated with the intake of LA (Pearson's correlation $=0 \cdot 42, P<0 \cdot 0001)$, but there was no correlation between the intake of ALA and that of $n-3$ LCPUFA (Pearson's correlation $=-0.005, P=0 \cdot 007)$.

Combined HR and 95\% CI for CHD events and deaths for an increase of $1 \mathrm{~g} / \mathrm{d}$ in the intake of ALA are given in Table 2 . There was a tendency towards a significant effect modification by sex for ALA $v$. CHD death ( $P$ for interaction=0.07). Among men, a non-significant inverse association was observed between the intake of ALA and the risk of CHD events and deaths. For each additional gram of ALA consumed, a $15 \%$

Table 2. Combined risk of CHD events and deaths for an additional intake of $1 \mathrm{~g} / \mathrm{d}$ of $\alpha$-linolenic acid (ALA) (Hazard ratios (HR) and $95 \%$ confidence intervals)*

\begin{tabular}{|c|c|c|c|c|c|c|c|}
\hline & \multicolumn{2}{|c|}{ All } & \multirow{2}{*}{$\begin{array}{l}P \text { for effect } \\
\text { modification } \\
\text { by sex }\end{array}$} & \multicolumn{2}{|c|}{ Women } & \multicolumn{2}{|c|}{ Men } \\
\hline & $\mathrm{HR}$ & $95 \% \mathrm{Cl}$ & & $\mathrm{HR}$ & $95 \% \mathrm{Cl}$ & $\mathrm{HR}$ & $95 \% \mathrm{Cl}$ \\
\hline \multicolumn{8}{|c|}{ ALA, CHD events } \\
\hline Model 1† & 0.94 & $0.82,1.08$ & 0.95 & 1.07 & $0.66,1.74$ & 0.95 & $0.86,1.05$ \\
\hline Model 2‡ & 0.93 & $0.84,1.04$ & 0.88 & 0.96 & $0.67,1.39$ & 0.95 & $0.86,1.05$ \\
\hline Model $3 \S$ & 0.88 & $0.75,1.02$ & 0.42 & 1.02 & $0.65,1.59$ & 0.85 & $0.72,1.01$ \\
\hline \multicolumn{8}{|c|}{ ALA, CHD deaths } \\
\hline Model 1† & 0.95 & $0.82,1.09$ & 0.62 & 1.04 & $0.64,1.68$ & 0.93 & $0.80,1.09$ \\
\hline Model $2 \ddagger$ & 0.96 & $0.83,1.11$ & 0.97 & 0.96 & $0.64,1.45$ & 0.96 & $0.82,1.13$ \\
\hline Model 3§ & 0.88 & $0.68,1 \cdot 14$ & 0.07 & 1.23 & $0.80,1.89$ & 0.77 & $0.58,1.01$ \\
\hline
\end{tabular}

* Data were analysed using Cox proportional hazards regression.

† Model 1 included energy-adjusted intake of ALA expressed in g/d. Age at baseline (years) and the calendar year in which the baseline questionnaire was returned were entered into the model through the STRATA statement.

$\ddagger$ Model 2 included the variables of model 1 and the following known risk factors for CHD: smoking habits; BMI; physical activity; educational level; history of hypertension.

$\S$ Model 3 included the variables of model 2 and the following dietary risk factors: alcohol intake; total energy intake (where alcohol is excluded); fibre intake; MUFA, SFA, trans-fatty acid, long-chain $n-3$ fatty acid, and linoleic acid intake. 

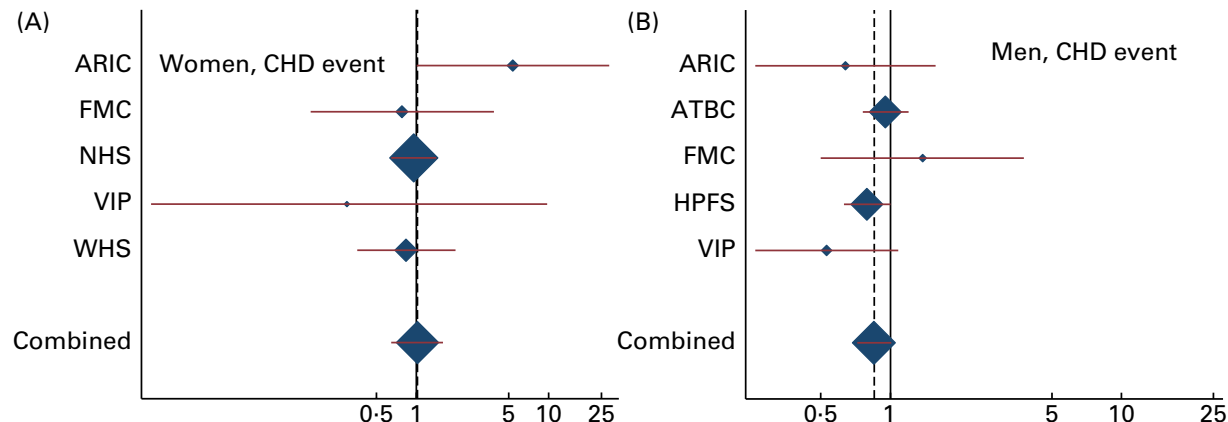

Fig. 1. Study-specific and combined risk of CHD events for each additional g/d intake of $\alpha$-linolenic acid in (A) women and (B) men in the Pooling Project of Cohort Studies on Diet and Coronary Disease. The multivariate model was adjusted for age at baseline and the calendar year in which the baseline questionnaire was returned, smoking habits, BMI, physical activity, educational level, history of hypertension, alcohol intake, total energy intake, fibre intake, and MUFA, trans-fatty acid, SFA, linoleic acid, and long-chain $n$-3 PUFA intake. Within each study, hazard ratios (HR) with $95 \% \mathrm{Cl}$ for the incidence of a CHD event and CHD death were calculated using Cox proportional hazards regression. Values are study-specific HR $(\diamond)$, with $95 \% \mathrm{Cl}$ represented by horizontal lines. The area of the - reflects the study-specific weight. The 'total ' represents the pooled HR and $95 \% \mathrm{Cl}$. (A) $P$ for heterogeneity=0.33; combined $\mathrm{HR} 1.02,95 \% \mathrm{Cl} 0.65,1.59$; (B) $P$ for heterogeneity $=0.37$; combined HR $0.85,95 \% \mathrm{Cl} 0.72,1.01$. ARIC, Atherosclerosis Risk in Communities Study; ATBC, Alpha-Tocopherol, Beta-Carotene Cancer Prevention Study; FMC, Finnish Mobile Clinic Health Examination Survey; HPFS, Health Professionals Follow-up Study; WHS, Women's Health Study; NHS, Nurses' Health Study; VIP, Västerbotten Intervention Program.

lower risk of CHD events (HR $0 \cdot 85,95 \% \mathrm{CI} 0 \cdot 72,1 \cdot 01)$ and a $23 \%$ lower risk of CHD deaths (HR $0.77,95 \%$ CI $0.58,1 \cdot 01$ ) were observed. Among women, no consistent association was observed between the intake of ALA and the risk of CHD events (HR 1.02, 95\% CI $0.65,1.59$ ) or CHD deaths (HR $1.23,95 \%$ CI $0.80,1.89$ ). Because the ARIC used the FFQ with only sixty-six items, we carried out an additional analysis without including the ARIC; however, this did not alter the findings (data not shown). The study-specific and combined HR and $95 \% \mathrm{CI}$ for CHD events and deaths for an increase of $1 \mathrm{~g} / \mathrm{d}$ in the intake of ALA are shown in Figs. 1 and 2 . The test for the presence of heterogeneity among the individual studies did not indicate any significant differences ( $P$ for heterogeneity among the studies were 0.33 and 0.72 for CHD events and deaths, respectively, among women and 0.37 and 0.39 for CHD events and deaths, respectively, among men). In analyses with ALA intake in quintiles, the lowest risk of CHD events was observed among those in the fifth quintile than in those in the first quintile among both women and men, but the differences were not significant (data shown in online supplementary Fig. S1). Among men, the lowest risk of CHD deaths was also observed in those in the fifth quintile than in those in the first quintile, but the differences were not significant (data shown in online supplementary Fig. S1). When the intake of ALA was evaluated as higher (>median) $v$. lower $(<$ median) among men, a significantly lower risk of CHD events was observed among men with an ALA intake value above the median $(1.17 \mathrm{~g} / \mathrm{d})$ than in those with an intake value below the median (HR $0.91, \quad 95 \%$ CI $0.83,1.00)$. No significant differences were observed among women when the intake of ALA was evaluated as higher (>median) $v$. lower $(<$ median) or among men when the outcome was CHD deaths (data given in Table S1, available online).

When assessing whether the association between ALA intake and CHD risk was modified by $n-3$ LCPUFA, the intake of ALA was found to be most strongly associated with the risk of CHD events among men (Fig. 3). However, none
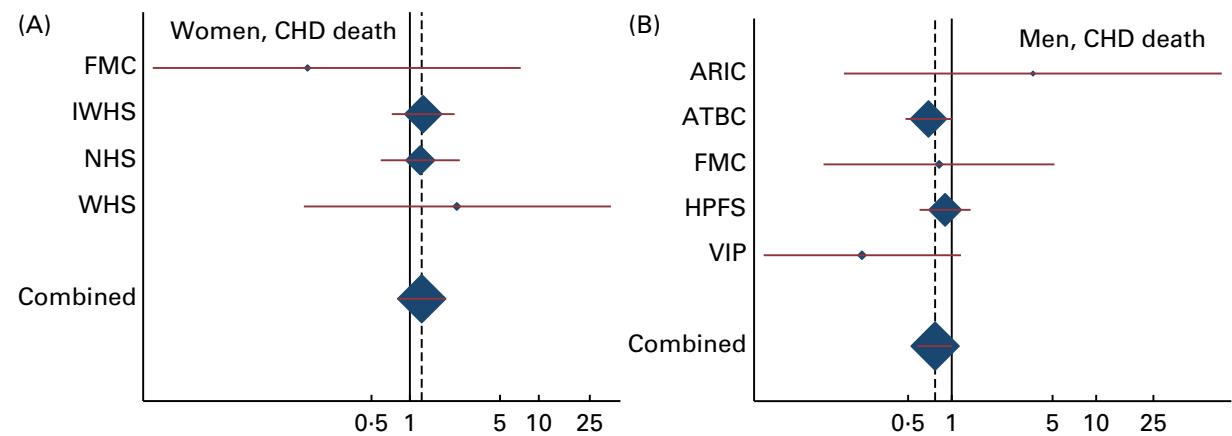

Fig. 2. Study-specific and combined risk of CHD death for each additional g/d intake of $\alpha$-linolenic acid in (A) women and (B) men in the Pooling Project of Cohort Studies on Diet and Coronary Disease. The multivariate model was adjusted for age at baseline and the calendar year in which the baseline questionnaire was returned, smoking habits, BMI, physical activity, educational level, history of hypertension, alcohol intake, total energy intake, fibre intake, and MUFA, trans-fatty acid, SFA, linoleic acid, and long-chain $n$-3 PUFA intake. Within each study, hazard ratios (HR) with $95 \% \mathrm{Cl}$ for the incidence of a CHD event and CHD death were calculated using Cox proportional hazards regression. Values are study-specific $\mathrm{HR}(\bullet)$, with $95 \% \mathrm{Cl}$ represented by horizontal lines. The area of the $\checkmark$ reflects the study-specific weight. The 'total $\bullet$ represents the pooled $\mathrm{HR}$ and $95 \% \mathrm{Cl}$. (A) $P$ for heterogeneity=0.72; combined $\mathrm{HR} 1.23,95 \% \mathrm{Cl} 0.80,1 \cdot 89$; (B) $P$ for heterogeneity=0.39; combined HR 0.77, $95 \% \mathrm{Cl} 0.58,1.01$. ARIC, Atherosclerosis Risk in Communities Study; ATBC, Alpha-Tocopherol, Beta-Carotene Cancer Prevention Study; FMC, Finnish Mobile Clinic Health Examination Survey; HPFS, Health Professionals Follow-up Study; IWHS, Iowa Women's Health Study; NHS, Nurses' Health Study; VIP, Västerbotten Intervention Program; WHS, Women's Health Study. 
(A)

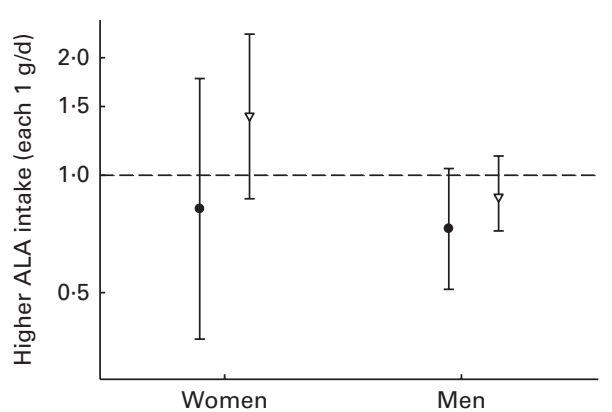

(B)

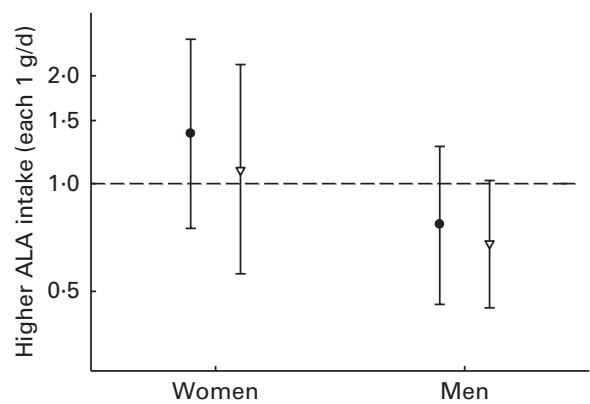

Fig. 3. Risk of (A) CHD events and (B) CHD deaths associated with each $1 \mathrm{~g} / \mathrm{d}$ of $\alpha$-linolenic acid (ALA) intake, among men and women with below (•) or above $(\nabla)$ median $n$-3 long-chain PUFA intake (women: $0.15 \mathrm{~g} / \mathrm{d}$; men: $0.26 \mathrm{~g} / \mathrm{d}$ ). Values are hazard ratios $(\mathrm{HR})$, with $95 \% \mathrm{Cl}$ represented by vertical bars. Data were analysed using Cox proportional hazards regression. The model was adjusted for age at baseline and the calendar year in which the baseline questionnaire was returned, smoking habits, BMI, physical activity, educational level, history of hypertension, alcohol intake, total energy intake, fibre intake, MUFA, trans-fatty acid, SFA, and linoleic acid intake. No significant differences were observed in women ( $\mathrm{A}) P=0.42$ and $(\mathrm{B}) P=0.43)$ and men $((\mathrm{A}) P=0.92$ and $(\mathrm{B}) P=0.79)$.

of the analyses revealed a significant association and no effect modification was observed. Carrying out analyses the same as those used to obtain data shown in Fig. 3, but with the cut-off values of $n-3$ LCPUFA of $0 \cdot 1 \mathrm{~g}$, suggested by Mozaffarian et $a l^{(14)}$, was not possible, due to the limited number of cases in the lowest-intake group. In interaction analysis with a common reference (low ALA intake and low $n$-3 LCPUFA intake), the lowest risk of CHD events was observed among women with a high intake of ALA intake and a low intake of $n-3$ LCPUFA (HR $0.83,95 \%$ CI $0.68,1.02 ; \quad P$ for trend=0.06) than among those with a low intake of both types of $n-3$ PUFA (Table S2, available online). Among men, the lowest risk of CHD was observed when both ALA and $n$-3 LCPUFA intakes were high. In age-stratified analyses, the intake of ALA was not significantly associated with the risk of CHD and no effect modification by age was observed (data not shown).

\section{Discussion}

In the present study, we found a non-significant inverse association between the dietary intake of ALA and the risk of incident CHD events and CHD deaths among men. The pooling of data from eight large cohort studies is a major strength of the present study and allowed studying the association between the intake of ALA and the risk of CHD among women and men in different populations consuming different diets and exhibiting a wide range of intakes. Variations in intake are essential for detecting potential associations with health. Furthermore, an advantage of using a pooling study design rather than a meta-analysis is that this design greatly reduces the risk of publication bias, because results originated both from studies that did not previously publish on the association between ALA intake and CHD risk and from studies that did. Indeed, only three of the eight studies included NHS $^{(26,32)}, \mathrm{ATBC}^{(28)}$ and $\mathrm{HPFS}^{(14,33)}$ ) had reported results from such an analysis earlier. Another strength of this pooled study is that data on the intake of ALA as well as covariates were harmonised and the same statistical approach was used across all the studies. Habitual dietary intake was determined using validated FFQ or by dietary history interviews. ALA levels in human tissue may provide a more accurate measure of habitual ALA intake than dietary records or questionnaires. However, ALA status in tissues may be influenced by metabolic differences between subjects with regard to genetics, sex and background diet, and day-to-day variations in tissue levels cannot be excluded either. The fact that our particular pooling study of prospective cohorts required an assumption of no changes in diet over time may also contribute to measurement error over time. Repeated measurements are required to reduce this, but these were available only for a small selected subset. Furthermore, the PUFA composition of foods, e.g. butter, have changed over time, meaning that even though people have used the same type and amount of butter during their lifetime, their fatty acid intakes have changed. This change is partly explained by the change in the practice of feeding ruminants with concentrated cereal grain-based feed rather than with grass, which is naturally rich in ALA. At the inception of the NHS and the HPFS in 1986, approximately $15-20 \%$ of ALA was derived from beef and dairy sources, but this percentage waned with follow-up.

Fatty acid compositions vary a lot in ready-made dishes and take-away foods, as well as in biscuits and cakes (depending on whether the fat used is of animal or plant origin). Furthermore, ALA (and fat in general) is difficult to be recognised and quantified in questionnaires and interviews, due to its role in food preparation - frying and cooking as well as a component in sauces and dressings. This could lead to random misclassification of ALA intake, which may result in the attenuation of associations. In the USA, e.g. in the NHS and the HPFS, the primary source of ALA is mayonnaise or other creamy dressings or oil and vinegar salad dressing, which may be difficult to be quantified using FFQ. Because salad dressings are major sources of ALA, ALA may be a proxy for vegetable intake; therefore, it could have been interesting to carry out stratified analyses on the intake of vegetables/salad in an attempt to remove confounding by the intake of vegetables/salad. We did not make any adjustment for vegetable/salad intake, because we did not have these data for all the cohorts 
included in the Pooling Project of Cohort Studies on Diet and Coronary Disease, and one principle of pooling is to treat all cohorts the same (same confounders). However, a previous study using data from only the NHS has made further adjustment for vegetable intake without any change in the results $^{(26)}$. However, the association could still have been influenced by a generally healthier lifestyle and diet of those who consumed more vegetables or salad. Another challenge in evaluating the association between ALA intake and CHD risk is that the intakes of LA and ALA are difficult to be differentiated, as they are often present in the same product, e.g. soyabean oil, margarine and mayonnaise. However, in the present study, the correlation coefficient was only $0 \cdot 42$, thus enabling statistical adjustment of LA intake in the multivariate analyses.

Several prospective cohort studies have evaluated the association between ALA intake and CHD risk and obtained inconsistent results ${ }^{(14,26,28,32-34)}$. Indeed, our findings are in line with those of a recent meta-analysis of thirteen prospective studies carried out in 2012 showing that higher ALA intakes (each $1 \mathrm{~g} / \mathrm{d}$ increment) are associated with a $10 \%$ lower risk of incident CVD (HR 0.90, 95\% CI 0.81, 0.99) ${ }^{(11)}$. However, in the evaluation of the association between the intake of ALA and the risk of CVD subtypes, CHD events and CHD deaths, only three studies were included in the meta-analysis of CHD events and six studies in the analysis of CHD deaths and the association was found to be significant only for ALA intake and CHD deaths (HR 0.80, 95\% CI 0.65, 0.98) (ALA and CHD events: HR 0.84, $95 \%$ CI 0.61, 1.15). Of these nine studies, four used cohorts that were also included in the present study (NHS, HPFS and ATBC). Only two of the nine studies were conducted in women (data from the NHS $)^{(26,32)}$ and six were conducted solely in men ${ }^{(28,33,35-38)}$, whereas the last study was conducted in a sample of both the sexes, with $27 \%$ women and $73 \%$ men $^{(39)}$. In the present study, we obtained different results for women and men. Whether the different results obtained for women and men reflect true biological differences or are due to power or are caused by some kind of bias remains to be determined. On the other hand, an inverse association between ALA intake and CHD risk is theoretically more likely among women than among men, as several studies have shown that the conversion of ALA into $n$ - 3 LCPUFA is stimulated by oestrogen and is greater in women ${ }^{(12,13)}$. The association between ALA intake and CHD risk has been evaluated by two prior studies in women, with both being carried out using data from the $\mathrm{NHS}^{(26,32)}$ and included in the recent meta-analysis by Pan et $a{ }^{(11)}$. In one of these studies, Hu et al. ${ }^{(26)}$ found that the intake of ALA is associated with a lower risk of CHD deaths (after 10 years of follow-up). In the other study, carried out by Albert et $a l^{(32)}$, analyses were carried out for both sudden death and overall CHD death and no association was found between the intake of ALA and the risk of total CHD deaths (after 18 years of follow-up). Albert et al. ${ }^{(32)}$ concluded that the beneficial effects of ALA intake on CHD deaths found by $\mathrm{Hu}$ et al. ${ }^{(26)}$ were probably due to its beneficial effects on sudden deaths, which account for a higher proportion of CHD deaths in younger women. Both these studies carried out using data from the NHS used a simple updated analysis that used the most recent ALA value available, which may explain the deviation of the results of the NHS in the present study. Furthermore, the age distribution in the present study was similar for both men and women, but the number of female cases was lower (death $=560$ and event $=1156$ ) than that of male cases (death $=1191$ and event $=3337$ ), because women, in general, are older when they experience CHD. Therefore, the estimate of an association between ALA intake and CHD risk among women may be statistically unclear, which may also explain the different results.

Mozaffarian et al. $^{(14)}$ showed that each additional $1 \mathrm{~g} / \mathrm{d}$ intake of ALA is associated with a $58 \%$ lower risk of CHD events (HR 0.42, 95\% CI 0.23, 0.75) among men with low $n$-3 LCPUFA intakes $(<0 \cdot 1 \mathrm{~g} / \mathrm{d})$ and is not associated with the risk of CHD events among men with higher $n$-3 LCPUFA intakes $(>0.1 \mathrm{~g} / \mathrm{d})(P$ for interaction $=0.0006)$. In the present study, among men with $n$-3 LCPUFA intakes below the median $(<0.26 \mathrm{~g} / \mathrm{d})$, each $1 \mathrm{~g} / \mathrm{d}$ of ALA intake was associated with a $27 \%$ lower risk of CHD events (HR 0.73, 95\% CI 0.51, $1 \cdot 04$ ), but among men with $n$-3 LCPUFA intakes above the median, no significant association was observed (HR 0.90, $95 \%$ CI $0.72,1 \cdot 12$ ) ( $P$ for interaction $=0.92$ ). Furthermore, no effect modification by the intake of $n$-3 LCPUFA was observed among women. Albert et al. ${ }^{(32)}$ have reported an additive effect of ALA and $n$-3 LCPUFA, indicating that the lowest risk of sudden cardiac deaths occurred among those with the highest intakes of both ALA and $n$ - 3 LCPUFA. In the present study, the lowest risk of CHD events and deaths was observed among men with high intakes of both ALA and $n$-3 LCPUFA, although it was not significant. However, the lowest risk of CHD events was observed among women with low ALA intakes and high $n$-3 LCPUFA intakes (HR $0.83,95 \%$ CI $0.68,1.02 ; P$ for trend $=0.06$ ) than among women with low intakes of both types of $n-3$ PUFA. Although CHD deaths and CHD events are both atherosclerotic disorders and have common risk factors, we found signs of differential effect modification by the ingestion of $n-3$ LCPUFA for the association between ALA intake and risk depending on whether the outcome was a CHD event or CHD death. However, ALA may be more important in individuals with low $n-3$ LCPUFA intakes because these individuals need ALA, whereas individuals with high $n-3$ LCPUFA intakes do not need it to achieve optimal levels of $n$-3 LCPUFA - so it may not be so much the feedback inhibition, but the deficiency of $n$-3 LCPUFA itself that matters. In our previous study in Danish men and women ${ }^{(40)}$, we found no effect modification by the ingestion of $n-3$ LCPUFA for the association between ALA intake and CHD risk.

\section{Conclusion}

In this large pooled study on the association between dietary ALA intake and CHD risk, an inverse association was observed among men; however, it was not significant. No consistent association was observed among women. This is the largest and most powerful study to evaluate the association between 
ALA intake and CHD risk to date. Given that a higher intake of ALA was found to reduce the risk of CHD, although only among men, from a public health point of view, it may be feasible to increase ALA intake. Rapeseed oil (which is rich in ALA) is the cheapest oil in many countries and thus can be used in household cooking by a majority of populations. However, rapeseed oil is best for raw use (i.e. salad dressing) and not for heating due to its degree of unsaturation. Other sources of ALA are, for instance, walnuts and flaxseeds, although their use is not very common in all populations. To obtain $1 \mathrm{~g}$ of ALA, one has to consume two teaspoons of rapeseed oil or $15 \mathrm{~g}$ of walnuts or $6 \mathrm{~g}$ of flaxseeds. Sex differences in prospective cohort studies have received little attention and deserve further investigation.

\section{Supplementary material}

To view supplementary material for this article, please visit http://dx.doi.org/10.1017/S000711451400138X

\section{Acknowledgements}

The authors are grateful to Alberto Ascherio and Aaron Folsom for their comments on the manuscript and involvement in the Pooling Project of Cohort Studies on Diet and Coronary Disease. They are also thankful to Gearóid O'Reilly for statistical assistance. They thank the staff and participants of the ARIC, the ATBC, the FMC, the HPFS, the IWHS, the NHS, the VIP and the WHS for their important contributions.

This work was carried out as a part of the research programme of the Danish Obesity Research Centre (DanORC). DanORC is supported by the Danish Council for Strategic Research (grant no. 2101-06-0005) and the National Institute of Health grants P01 CA87969 (NHS), UM1 CA167552 (HPFS) and R01 HL58904.

The authors' contributions are as follows: M. S. V., M. U. J., L. L. and B. L. H. contributed to the study concept and design; M. S. V., M. U. J., L. L., B. L. H., G. H., G. C., J. B., P. K., J. V., L. M. S., K. R., E. B. R. and E. J. O. interpreted the data and critically revised the manuscript; $M$. S. V. drafted the manuscript; M. S. V. and E. J. O. carried out the statistical analyses.

None of the authors has any conflicts of interest to declare.

\section{References}

1. WHO (2011) World Heart Federation, World Stroke Organization. Global Atlas on Cardiovascular Disease Prevention and Control. Geneva: WHO.

2. Bang HO, Dyerberg J \& Hjoorne N (1976) The composition of food consumed by Greenland Eskimos. Acta Med Scand 200, 69-73.

3. Hansen SN \& Harris WS (2007) New evidence for the cardiovascular benefits of long chain omega-3 fatty acids. Curr Atheroscler Rep 9, 434-440.

4. Harris WS, Miller M, Tighe AP, et al. (2008) Omega-3 fatty acids and coronary heart disease risk: clinical and mechanistic perspectives. Atherosclerosis 197, 12-24.
5. Harris WS, Kris-Etherton PM \& Harris KA (2008) Intakes of long-chain omega-3 fatty acid associated with reduced risk for death from coronary heart disease in healthy adults. Curr Atheroscler Rep 10, 503-509.

6. Mozaffarian D \& Wu JH (2011) Omega-3 fatty acids and cardiovascular disease: effects on risk factors, molecular pathways, and clinical events. J Am Coll Cardiol 58, 2047-2067.

7. Delgado-Lista J, Perez-Martinez $\mathrm{P}$, Lopez-Miranda J, et al. (2012) Long chain omega-3 fatty acids and cardiovascular disease: a systematic review. Br J Nutr 107, Suppl. 2, S201-S213.

8. Brouwer IA, Katan MB \& Zock PL (2004) Dietary $\alpha$-linolenic acid is associated with reduced risk of fatal coronary heart disease, but increased prostate cancer risk: a meta-analysis. J Nutr 134, 919-922.

9. Wang C, Harris WS, Chung M, et al. (2006) n-3 Fatty acids from fish or fish-oil supplements, but not $\alpha$-linolenic acid, benefit cardiovascular disease outcomes in primaryand secondary-prevention studies: a systematic review. Am J Clin Nutr 84, 5-17.

10. Geleijnse JM, de Goede J \& Brouwer IA (2010) $\alpha$-Linolenic acid: is it essential to cardiovascular health? Curr Atheroscler Rep 12, 359-367.

11. Pan A, Chen M, Chowdhury R, et al. (2012) $\alpha$-Linolenic acid and risk of cardiovascular disease: a systematic review and meta-analysis. Am J Clin Nutr 96, 1262-1273.

12. Burdge $G$ (2004) $\alpha$-Linolenic acid metabolism in men and women: nutritional and biological implications. Curr Opin Clin Nutr Metab Care 7, 137-144.

13. Decsi $T \&$ Kennedy K (2011) Sex-specific differences in essential fatty acid metabolism. Am J Clin Nutr 94, Suppl. 6, 1914S-1919S.

14. Mozaffarian D, Ascherio A, Hu FB, et al. (2005) Interplay between different polyunsaturated fatty acids and risk of coronary heart disease in men. Circulation 111, 157-164.

15. Fraser GE, Strahan TM, Sabate J, et al. (1992) Effects of traditional coronary risk factors on rates of incident coronary events in a low-risk population. The Adventist Health Study. Circulation 86, 406-413.

16. The ARIC Investigators (1989) The Atherosclerosis Risk in Communities (ARIC) Study: design and objectives. The ARIC investigators. Am J Epidemiol 129, 687-702.

17. The ATBC Cancer Prevention Study Group (1994) The alpha-tocopherol, beta-carotene lung cancer prevention study: design, methods, participant characteristics, and compliance. The ATBC Cancer Prevention Study Group. Ann Epidemiol 4, 1-10

18. Knekt P, Reunanen A, Jarvinen R, et al. (1994) Antioxidant vitamin intake and coronary mortality in a longitudinal population study. Am J Epidemiol 139, 1180-1189.

19. Schroll M, Jorgensen T \& Ingerslev J (1992) The Glostrup Population Studies, 1964-1992. Dan Med Bull 39, 204-207.

20. Rimm EB, Giovannucci EL, Stampfer MJ, et al. (1992) Reproducibility and validity of an expanded self-administered semiquantitative food frequency questionnaire among male health professionals. Am J Epidemiol 135, 1114-1126.

21. Goldbourt U \& Yaari S (1990) Cholesterol and coronary heart disease mortality. A 23-year follow-up study of 9902 men in Israel. Arteriosclerosis 10, 512-519.

22. Folsom AR, Kushi LH, Anderson KE, et al. (2000) Associations of general and abdominal obesity with multiple health outcomes in older women: the Iowa Women's Health Study. Arch Intern Med 160, 2117-2128. 
23. Willett WC, Sampson L, Stampfer MJ, et al. (1985) Reproducibility and validity of a semiquantitative food frequency questionnaire. Am J Epidemiol 122, 51-65.

24. Winkvist A, Hornell A, Hallmans G, et al. (2009) More distinct food intake patterns among women than men in northern Sweden: a population-based survey. Nutr J 8, 12.

25. Lee IM, Cook NR, Gaziano JM, et al. (2005) Vitamin E in the primary prevention of cardiovascular disease and cancer: the Women's Health Study: a randomized controlled trial. JAMA 294, 56-65.

26. Hu FB, Stampfer MJ, Manson JE, et al. (1999) Dietary intake of $\alpha$-linolenic acid and risk of fatal ischemic heart disease among women. Am J Clin Nutr 69, 890-897.

27. Pietinen P, Hartman AM, Haapa E, et al. (1988) Reproducibility and validity of dietary assessment instruments. I. A self-administered food use questionnaire with a portion size picture booklet. Am J Epidemiol 128, 655-666.

28. Pietinen P, Ascherio A, Korhonen P, et al. (1997) Intake of fatty acids and risk of coronary heart disease in a cohort of Finnish men: the Alpha-Tocopherol, Beta-Carotene Cancer Prevention Study. Am J Epidemiol 145, 876-887.

29. Mizrahi A, Knekt P, Montonen J, et al. (2009) Plant foods and the risk of cerebrovascular diseases: a potential protection of fruit consumption. Br J Nutr 102, 1075-1083.

30. Pereira MA, O'Reilly E, Augustsson K, et al. (2004) Dietary fiber and risk of coronary heart disease: a pooled analysis of cohort studies. Arch Intern Med 164, 370-376.

31. Jakobsen MU, O'Reilly EJ, Heitmann BL, et al. (2009) Major types of dietary fat and risk of coronary heart disease: a pooled analysis of 11 cohort studies. Am J Clin Nutr 89, $1425-1432$.
32. Albert CM, Oh K, Whang W, et al. (2005) Dietary $\alpha$-linolenic acid intake and risk of sudden cardiac death and coronary heart disease. Circulation 112, 3232-3238.

33. Ascherio A, Rimm EB, Giovannucci EL, et al. (1996) Dietary fat and risk of coronary heart disease in men: cohort follow up study in the United States. BMJ 313, 84-90.

34. de Goede J, Verschuren WM, Boer JM, et al. (2011) $\alpha$-Linolenic acid intake and 10 -year incidence of coronary heart disease and stroke in 20,000 middle-aged men and women in the Netherlands. PLOS ONE 6, e17967.

35. Dolecek TA \& Granditis G (1991) Dietary polyunsaturated fatty acids and mortality in the Multiple Risk Factor Intervention Trial (MRFIT). World Rev Nutr Diet 66, 205-216.

36. Oomen CM, Ocke MC, Feskens EJ, et al. (2001) $\alpha$-Linolenic acid intake is not beneficially associated with $10-\mathrm{y}$ risk of coronary artery disease incidence: the Zutphen Elderly Study. Am J Clin Nutr 74, 457-463.

37. Laaksonen DE, Nyyssonen K, Niskanen L, et al. (2005) Prediction of cardiovascular mortality in middle-aged men by dietary and serum linoleic and polyunsaturated fatty acids. Arch Intern Med 165, 193-199.

38. Lopes C, Aro A, Azevedo A, et al. (2007) Intake and adipose tissue composition of fatty acids and risk of myocardial infarction in a male Portuguese community sample. $J \mathrm{Am}$ Diet Assoc 107, 276-286.

39. Campos H, Baylin A \& Willett WC (2008) $\alpha$-Linolenic acid and risk of nonfatal acute myocardial infarction. Circulation 118, 339-345.

40. Vedtofte MS, Jakobsen MU, Lauritzen L, et al. (2011) Dietary $\alpha$-linolenic acid, linoleic acid, and $n-3$ long-chain PUFA and risk of ischemic heart disease. Am J Clin Nutr 94 , $1097-1103$. 